\title{
Using Unsupervised Artificial Neural Networks to Detect Sibling Species: A case in Myxomycetes
}

\author{
Francisco Pando $\ddagger$, Ignacio Heredia ${ }^{\S}$, Lara Lloret ${ }^{\S}$ \\ ‡ Real Jardin Botanico -CSIC, Madrid, Spain \\ $\S$ Insituto de Fisica de Cantabria - CSIC/UC, Santander, Spain
}

Corresponding author: Francisco Pando (pando@rib.csic.es)

Received: 13 Jun 2019 | Published: 19 Jun 2019

Citation: Pando F, Heredia I, Lloret L (2019) Using Unsupervised Artificial Neural Networks to Detect Sibling Species:

A case in Myxomycetes. Biodiversity Information Science and Standards 3: e37255.

https://doi.org/10.3897/biss.3.37255

\section{Abstract}

Species distribution modelling (SDM) --i.e. the prediction of species potential geographic distributions based on correlations between known presence records and the environmental conditions at occurrence localities-- is one of the most freqently cited developments in recent years in the realm of biodiversity studies (Web of Science 2019). The reasons for the explosion in SDM studies reside in: a) the pressure to know species distributions (under both present and future climate change scenarios) with precision to satisfy scientific as well as societal objectives; b) the impossibility of knowing every occurrence of all but the most conspicuous and/or special interest species; c) the availability of primary occurrence and environmental data in unprecedented amounts, thanks to initiatives like the Global Biodiversity Information Facility (GBIF); and d) the development of algorithms and software, along with the computing power, which allow inference of species distribution models from the available data.

The standard methods to produce such models are based on environmental feature vectors, and some well-established algorithms such as distance-based machine learning, regression or a combination of these (Tsoar et al. 2007).

In this presentation, we explore deep learning techniques (LeCun et al. 2015), particularly how those developed in recent years could contribute to the study of species distribution (see also Botella et al. 2018; Deneu et al. 2018). In this contribution we aim to 
identify sibling species on the basis of their ecological preferences. In this exercise, we prepare an image-based environmental representation space using an unsupervised classification approach, instead of a set of environmental feature vectors. For our case study, we chose a well-known cosmopolitan myxomycete (i.e., mycetozoan) species: Hemitrichia serpula (Scop.) Rostaf., whose ecological preferences --involving several biomes-- may suggest that it could comprise several sibling species.

\section{Keywords}

deep learning, species distribution, ecological niche modelling, sibling species, Myxomycetes

\section{Presenting author}

Francisco Pando

\section{Presented at}

Biodiversity_Next 2019

\section{Funding program}

This project has received funding from the European Union's Horizon 2020 research and innovation programme under grant agreement No 777435

\section{References}

- $\quad$ Botella C, Joly A, Bonnet P, Monestiez P, Munoz F (2018) A Deep Learning Approach to Species Distribution Modelling. In: Joly A, Vrochidis S, Karatzas K, Karppinen A, Bonnet P (Eds) Multimedia Tools and Applications for Environmental \& Biodiversity Informatics. Multimedia Systems and Applications. Springer, Cham. [ISBN 978-3-319-76445-0]. https:// doi.org/10.1007/978-3-319-76445-0 10

- Deneu B, Servajean M, Botella C, Joly A (2018) Location-based species recommendation using co-occurrences and environment-GeoLifeCLEF 2018 challenge. Conference and Labs of the Evaluation Forum, CEUR Workshop Proceedings. CLEF: Conference and Labs of the Evaluation Forum Working Notes of CLEF, Sep. 2018. Avignon, France, 14 pp. URL: https://hal.archives-ouvertes.fr/hal-01913241

- $\quad$ LeCun Y, Bengio Y, Hinton G (2015) Deep learning. Nature 521 (7553): 436-444. https:// doi.org/10.1038/nature14539 
- Tsoar A, Allouche O, Steinitz O, Rotem D, Kadmon R (2007) A comparative evaluation of presence-only methods for modelling species distribution. Diversity and Distributions 13 (4): 397-405. https://doi.org/10.1111/j.1472-4642.2007.00346.x

- Web of Science (2019) TS=(niche model) OR TS=(distribution model) AND (WC=( ENVIRONMENTAL SCIENCES $)$ OR WC $=($ ENVIRONMENTAL SCIENCES OR ECOLOGY ) OR WC $=($ ECOLOGY $)$ OR WC $=($ ECOLOGY OR ENVIRONMENTAL SCIENCES OR BIODIVERSITY CONSERVATION OR AGRICULTURE MULTIDISCIPLINARY)). https:// tinyurl.com/SDM-explosion. Accessed on: 2019-4-30. 